\title{
Tracheomalacia and bronchomalacia in children: response to the ERS statement
}

\author{
To the Editor:
}

We welcome the timely publication of the European Respiratory Society statement on tracheomalacia and bronchomalacia (TBM) in children [1], but are concerned that the definition of malacia used in the statement may contribute to underdiagnosis in those older children with milder degrees of malacia whose main symptom is exercise limitation [2]. Malacia in the statement is defined as an "arbitrary $>50 \%$ expiratory reduction in the cross-sectional luminal area during quiet respiration" diagnosed with the use of multidetector computed tomography (MDCT); the authors cite seven references in support of their definition. However, these references include: 15 children who were asked to "take a deep breath and then blow all the way out and hold it" [3]; 18 patients aged 1 month to 17 years whereby those over 5 years were asked to "blow their breath out and hold it" [4]; a description of protocol where the authors state that "dynamic expiratory and coughing manoeuvres are generally more sensitive for detecting malacia than end expiratory manoeuvres" and "we may ask the child to perform repetitive coughs in a supine position in the scanner" [5]; a reduction in tracheal cross-sectional area of $>31.6 \%$ as being diagnostic [6]; and, finally, 23 infants all of whom were intubated and where malacia was defined as a reduction in airway cross-sectional area $>28 \%$ based on data from 10 adults with a mean age of 60 years with acquired TBM diagnosed using forced expiratory manoeuvres $[7,8]$. In other words, the protocols in these studies made use of dynamic expiration rather than quiet respiration when the child was older than 5 years, was not sedated and was not intubated. During quiet breathing in children with normal airways, intraluminal airway pressure does not fall below pleural pressure and airway collapse does not occur. In children with milder degrees of malacia, a clinically significant reduction in cross-sectional airway area may not be evident during quiet breathing and may occur only when pleural pressure is substantially greater than intraluminal pressure as is the case during forced expiration or with cough [5]. It is likely that there is overlap in the degree of collapsibility that is observed in the airways of healthy children and those with TBM such that the significance of the degree of airway collapse observed should be interpreted in light of the patient's symptoms and signs, associated risk factors and lung function [5]. The authors state that manoeuvres such as exercise, coughing, crying, feeding, forced expiration and the Valsalva manoeuvre may aggravate malacia symptoms, but in older children with milder degrees of malacia, their symptoms may be apparent only during these manoeuvres [9]. We suggest that controlled dynamic expiratory manoeuvres may be more informative than quiet respiration when using MDCT or magnetic resonance imaging as a substitute for flexible bronchoscopy in older, cooperative children undergoing investigation for suspected TBM [10], or with symptoms of impaired exercise tolerance and asthma unresponsive to treatment [2].

@ERSpublications

Dynamic expiratory manoeuvres should be used to diagnose mild airway malacia http://bit.ly/2NBJ3Mn

Cite this article as: Crowley S, Carlsen K-H. Tracheomalacia and bronchomalacia in children: response to the ERS statement. Eur Respir J 2019; 54: 1902098 [https://doi.org/10.1183/13993003.02098-2019].

Suzanne Crowley ${ }^{1}$ and Kai-Håkon Carlsen ${ }^{2}$

${ }^{1}$ Paediatric Dept of Allergy and Lung Diseases, Oslo University Hospital, Oslo, Norway. ${ }^{2}$ Faculty of Medicine, University of Oslo, Oslo, Norway.

Correspondence: Suzanne Crowley, Paediatric Dept of Allergy and Lung Diseases, Oslo University Hospital, Sognsvannsveien 20, Oslo, Norway. E-mail: suzanne.crowley@gmail.com

Received: 27 Oct 2019 | Accepted: 31 Oct 2019

Conflict of interest: S. Crowley has nothing to disclose. K-H. Carlsen has nothing to disclose.

\section{References}

1 Wallis C, Alexopoulou E, Antón-Pacheco JL, et al. ERS statement on tracheomalacia and bronchomalacia in children. Eur Respir J 2019; 54: 1900382. 
2 Boogaard R, Huijsmans SH, Pijnenburg MWH, et al. Tracheomalacia and bronchomalacia in children: incidence and patient characteristics. Chest 2005; 128: 3391-3397.

3 Lee EY, Zurakowski D, Waltz DA, et al. MDCT evaluation of the prevalence of tracheomalacia in children with mediastinal aortic vascular anomalies. J Thorac Imaging 2008; 23: 258-265.

4 Ngerncham M, Lee EY, Zurakowski D, et al. Tracheobronchomalacia in pediatric patients with esophageal atresia: comparison of diagnostic laryngoscopy/bronchoscopy and dynamic airway multidetector computed tomography. J Pediatr Surg 2015; 50: 402-407.

5 Lee EY, Boiselle PM. Tracheobronchomalacia in infants and children: multidetector CT evaluation. Radiology 2009; 252: 7-22.

6 Goo HW. Free breathing cine CT for the diagnosis of tracheomalacia in young children. Pediatr Radiol 2013; 43: 922-928.

7 Greenberg SB, Dyamenahalli U. Dynamic pulmonary computed tomography angiography: a new standard for evaluation of combined airway and vascular abnormalities in infants. Int J Cardiovasc Imaging 2014; 30: 407-414.

8 Aquino S, Shepard JA, Ginns LC, et al. Acquired tracheomalacia: detction by expiratory CT scan. J Comput Assist Tomogr 2001; 25: 394-399.

$9 \quad$ Finder JD. Primary bronchomalacia in infants and children. J Pediatr 1997; 130: 59-66.

10 Ciet P, Wielopolski P, Manniesing R, et al. Spirometer-controlled cine magnetic resonance imaging used to diagnose tracheobronchomalacia in paediatric patients. Eur Respir J 2014; 43: 115-124.

Copyright @ERS 2019

From the authors:

We thank S. Crowley and K-H. Carlsen for their interest in the European Respiratory Society task force document on tracheomalacia and bronchomalacia (TBM) in children [1].

CrossMark

We agree that the definition of TBM is far from straightforward. The task force had to settle on a definition of malacia based on a systematic review of the literature combined with expert opinions from various parts of the world. The aim of the task force was to create a standard against which further work can be built. This is a working definition to advance the field as opposed to a single, poorly accepted, strict definition.

Many definitions of TBM in both children and adults exist, including those based only on a shape abnormality without any regard to a reduction in cross-sectional area [2]. Some include excessive dynamic airway collapse (EDAC) in the definition but others exclude EDAC to prevent overdiagnosis [3, 4]. As such, quiet breathing rather than forced coughing or Valsalva efforts in children is suggested to establish the baseline dynamic structure. The manoeuvre described by S. Crowley and K-H. Carlsen is likely to reveal EDAC rather than true cartilaginous tracheomalacia, although it is known that there is an overlap. Even in adult medicine where there is substantially more data, such controversy exists [5].

The authors correctly state that the controlled dynamic expiratory manoeuvres can only be done in older cooperative children. We were also mindful of the need in this paediatric statement to ensure that we did not generate false-positive findings in infants where the issues of malacia are particularly prevalent [6].

If dynamic expiratory studies are done in older children, investigators will need to consider if this is showing structural abnormities to the rings or EDAC. The subsequent correlation of these findings to the clinical symptoms is, of course, another story.

Colin Wallis ${ }^{1}$, Kostas Priftis ${ }^{2}$, Anne Chang ${ }^{3,4,5}$, Fabio Midulla ${ }^{6}$ and Jayesh Bhatt $\overbrace{}^{7}$

${ }^{1}$ Respiratory Medicine Unit, Great Ormond Street Hospital for Children, London, UK. ${ }^{2}$ Dipartimento Salute della Donna e del Bambino, Università degli Studi di Padova, Padova, Italy. ${ }^{3}$ Dept of Respiratory and Sleep Medicine, Queensland Children's Hospital, Brisbane, Australia. ${ }^{4}$ Centre for Children's Health Research, Institute of Health and Biomedical Innovation, Queensland University of Technology, Brisbane, Australia. ${ }^{5}$ Child Health Division, Menzies School of Health Research, Charles Darwin University, Casuarina, Australia. ${ }^{6}$ Dept of Paediatrics, "Sapienza" University of Rome, Rome, Italy. ${ }^{7}$ Nottingham University Hospitals NHS Trust, Queen's Medical Centre, Nottingham, UK.

Correspondence: Colin Wallis, Respiratory Medicine Unit, Great Ormond Street Hospital for Children, Great Ormond Street, London, WC1N 3JH, UK. E-mail: colin.wallis@gosh.nhs.uk

@ERSpublications

A working definition for tracheobronchomalacia in children is provided in the task force document published by the ERS in 2019. This may require review in due course as further research into the dynamic aspects of malacia and clinical correlation emerges. http://bit.ly/2rNuq08

Cite this article as: Wallis C, Priftis K, Chang A, et al. Tracheomalacia and bronchomalacia in children: response to the ERS statement. Eur Respir J 2019; 54: 1902271 [https://doi.org/10.1183/13993003.022712019]. 
Received: 25 Nov 2019 | Accepted: 26 Nov 2019

Conflict of interest: C. Wallis has nothing to disclose. K. Priftis has nothing to disclose. A. Chang received grants from National Health and Medical Research Council, Australia, has participated on a data monitoring committee for GSK, and has authored work for Up to Date and BMJ Evidence Centre, outside the submitted work. F. Midulla has nothing to disclose. J. Bhatt reports personal fees for lectures from Vertex, outside the submitted work.

\section{References}

1 Wallis C, Alexopoulou E, Antón-Pacheco JL, et al. ERS statement on tracheomalacia and bronchomalacia in children. Eur Respir J 2019; 54: 1900382.

2 Masters IB. Congenital airway lesions and lung disease. Pediatr Clin N Am 2009; 56: 227-242.

Murgu SD, Colt HG. Tracheobronchomalacia: untangling the Gordian knot. J Bronchol 2005; 12: 239-244.

4 Murgu SD, Colt HG. Tracheobronchomalacia and excessive dynamic airway collapse. Respirology 2006; 11: 388-406.

5 Murgu S, Stoy S. Excessive dynamic airway collapse: a standalone cause of exertional dyspnea? Ann Am Thorac Soc 2016; 13: 1437-1439.

6 Masters IB, Chang AB, Patterson L, et al. Series of laryngomalacia, tracheomalacia, and bronchomalacia disorders and their associations with other conditions in children. Pediatr Pulmonol 2002; 34: 189-195.

Copyright @eERS 2019 